\title{
Resistance to HER2-Targeted Therapies Results in Upregulation of MCL-1 and Sensitivity to Olaparib§
}

\author{
Andrew Armstrong ${ }^{1}$, Matthew Najor ${ }^{2}$, Trevor Rempert ${ }^{1}$, \\ Faraz Bishehsari ${ }^{1}$, Abde M. Abukhdeir ${ }^{2}$, Melody A. Cobleigh ${ }^{2}$
}

\author{
${ }^{1}$ Department of Medicine, Division of Gastroenterology, Rush University Medical Center \\ ${ }^{2}$ Department of Medicine, Division of Hematology, Oncology, and Cell Therapy, Rush University Medical Center
}

\begin{abstract}
${ }^{\S}$ The work herein was completed while AMA was a faculty member at Rush University. AMA is currently an employee with the U.S. Food and Drug Administration. The views and data in this publication do not reflect the opinions of the U.S. Government or The U.S. Food and Drug Administration.
\end{abstract}

Funding: This study was funded by Bears Care.

Conflicts of Interest: All authors declare that they have no conflicts of interest.

Authors' Contributions: All authors contributed to the study conception and design. Material preparation, data collection, and analysis were performed by AA, MN, TR, AMA. All authors have read and approved the final manuscript.

Data Availability: All data is available upon request.

\begin{abstract}
HER2 amplification results in increased tumor growth and aggressiveness in breast cancer. Despite advancements in HER2-targeted therapy, treatment resistance, cancer recurrence, and metastasis remain significant obstacles in combating this deadly disease. PARP inhibitors, have emerged as a promising class of drugs targeting cancers deficient in homologous recombination repair. Recent preclinical evidence suggests that PARP inhibitors exhibit sensitivity in cancers with high replication stress and genomic instability, as well as some HER2 positive breast cancer cell lines. To investigate the relationship between HER2 amplification and PARP inhibitor sensitivity, we utilized isogenic models of HER2-positive breast cancer, whereby cells were either sensitive or resistant to lapatinib and treated with the PARP inhibitor olaparib used as a single agent, or in combination with HER2-targeted therapies. Our results show that HER2 overexpressing MCF-10A cells are highly sensitivity to single-agent olaparib. The addition of trastuzumab and lapatinib resulted in a synergistic increase in toxicity. To determine if olaparib could be used to overcome resistance to lapatinib, we obtained SKBR3 cells conditioned to be resistant to lapatinib. Despite resistance to lapatinib, these cells continue to exhibit sensitivity to olaparib. Reverse phase protein analysis revealed that lapatinib resistant clones exhibited a statistically-significant increase in protein expression of the apoptosis inhibitor MCL-1, which is decreased upon olaparib treatment resulting in apoptosis. Taken together, our findings suggest that HER2 overexpression may predict sensitivity to olaparib alone, or in combination with trastuzumab and lapatinib. Lapatinib resistant HER2-positive breast cancer cells are sensitive to olaparib, possibly through downregulation of MCL-1.
\end{abstract}

Key Points: (1) Overexpression of HER2 in breast epithelial cells renders them susceptible to the PARP inhibitor olaparib, which synergizes with HER2 targeted agents to induce cytotoxicity. (2) Resistance to lapatinib may involve upregulation of the cell protective protein MCL-1, which is decreased with olaparib treatment and results in cell death.

\section{Introduction}

Epidermal growth factor receptor 2 (HER2) is a transmembrane tyrosine kinase that belongs to the epidermal growth factor receptor (EGFR) family of receptor tyrosine kinases. Amplification or overexpression of the HER2 oncogene occurs in some solid tumors, including about $20-25 \%$ of breast cancers [1]. HER2 overexpression is associated with increased tumor aggressiveness [2]. HER2 signaling activates signal transduction pathways associated with epidermal cell growth and differentiation. Several treatment strategies have been developed to target HER2, including antibodies that target the extracellular domain (trastuzumab, pertuzumab), and small molecule inhibitors that target the tyrosine kinase domain (lapatinib, tucatinib). Despite these

This article is published under the terms of the Creative Commons Attribution License 4.0

Author(s) retain the copyright of this article. Publication rights with Alkhaer Publications.

Published at: http://www.ijsciences.com/pub/issue/2020-12/

DOI: 10.18483/ijSci.2407; Online ISSN: 2305-3925; Print ISSN: 2410-4477 
targeted treatment options, treatment resistance, cancer recurrence, and metastasis remain significant obstacles in the treatment of this disease [3, 4]. Identifying new treatment strategies is necessary to address these obstacles.

PARP inhibitors (PARPi) are a class of drugs that target members of the Poly(ADP-ribose) Polymerase (PARP) protein family. PARP proteins function as DNA damage sensors and signal transductors for the nucleotide excision repair (NER) pathway. The NER pathway is integral to the repair of single strand breaks. PARP proteins bind to areas of DNA damage and undergo PARlaytion, a process that initiates the recruitment of DNA damage repair proteins to sites of DNA damage. PARPi have proven to be effective treatment options for cancers deficient in homologous recombination (HR) DNA repair [5]. Cancers deficient in HR often rely on alternative methods of DNA damage repair for sustained proliferation. In these cells, inhibition of alternative DNA repair pathways, such as NER, can induce catastrophic DNA damage and apoptosis, a form of synthetic lethality [6]. Apart from the established role in preventing PARlaytion, PARPi also trap PARP protein complexes to areas of DNA damage. Unresolved PARP-DNA complexes result in stalling and collapse of DNA replication forks resulting in double strand breaks [7]. Recent reports have revealed that cancers with high genomic instability and replication stress may be sensitive to PARP inhibition, possibly through catastrophic DNA damage as a result of PARP trapping [6].

Overexpression of HER2 drives proliferation and has been linked to genomic instability and a high frequency of chromosomal alterations [8]. These unique characteristics of HER2 signaling make HER2 positive breast cancers attractive targets for studying the utility of PARPi. In addition, some preclinical evidence suggests that HER2 breast cancers cells lines are sensitive to PARPi [9-13], and a phase II clinical trial is currently evaluating the safety and efficacy of olaparib in combination with trastuzumab for the treatment of BRCA-mutated or homologous recombination deficient advanced breast cancers [14]. This study supports the investigation of PARP inhibitors in cancers with high replication stress and the clinical utility of PARP inhibitors in combination with HER2 targeted therapies.

\section{Material and Methods \\ Cell Lines and Reagents}

The MCF-10A (ATCC, Manassas, VA) cell lines and its derivatives were cultured in Dulbecco's modified eagle medium (DMEM:F12) devoid of phenol-red (Life Technologies, Grand Island, NY) supplemented with 5\% horse serum (Sigma, Saint Louis, MO), $1 \%$ penicillin and streptomycin (Life Technologies,
Grand Island, NY), $20 \mathrm{ng} / \mathrm{mL}$ epidermal growth factor (EGF) (Sigma, Saint Louis, MO), $25 \mu \mathrm{g} / \mathrm{mL}$ insulin (Sigma, Saint Louis, MO), $0.5 \mu \mathrm{g} / \mathrm{mL}$ hydrocortisone (Sigma, Saint Louis, MO), and $0.1 \mu \mathrm{g} / \mathrm{mL}$ cholera toxin (Sigma, Saint Louis, MO) as previously described [15]. MCF-10A clones expressing HER2 were cultured in EGF-free supplemented medium containing $0.4 \mu \mathrm{M}$ puromycin. All assays of MCF-10A cells and its derivatives were performed in supplemented medium, whereby horse serum was replaced with $1 \%$ charcoal-dextran treated fetal bovine serum (CD-FBS) (Fisher Scientific, Pittsburg, PA) supplemented with $0.2 \mathrm{ng} / \mathrm{mL}$ EGF (hereafter referred to as "assay medium"). SKBR3 cells and their derivatives were grown in DMEM supplemented with $1 \%$ penicillin and streptomycin, and 10\% FBS. SKBR3-LR and parental cells were a generous gift from Dr. William Han (Dana-Faber Cancer Institute). Olaparib and lapatinib were obtained from Selleck Chemical. Trastuzumab was obtained from Rush University Medical Center.

\section{Cell Proliferation Assays}

Cells were plated in 96-well plates at a density of 500-1000 cells/well in assay medium. After 24 hours (day 1), the medium was replenished with fresh assay medium supplemented with $0.2 \mathrm{ng} / \mathrm{mL}$ EGF and drugs as indicated. After 72 hours (day 4), cells were stained with either crystal violet [15] or CellTiterFluor cell viability assay (Promega, Madison, WI) following the manufacturer's instructions and counted by measuring absorbance on a SpectraMax M5 fluorescence plate reader (Molecular Devices, Sunnydale, CA). Processing of raw absorbance data was performed using Microsoft Excel. Statistical analysis was performed using a two-way ANOVA GraphPad Prism 8. Three dimensional plots were generated using the Plot3d and Stats R packages [16, 17]. Synergy plots and significance was generated using the synergyfinder R package [18].

\section{Reverse Phase Protein Array}

Reverse Phase Protein Array was performed by MD Anderson Cancer Center (MDACC), and protein lysates were prepared using a protocol and reagents provided by MDACC. Cell lysate samples were serially diluted two-fold for 5 dilutions (undiluted, $1: 2,1: 4,1: 8 ; 1: 16)$ and arrayed on nitrocellulosecoated slides in an $11 \times 11$ format to produce sample spots. Sample spots were then probed with antibodies by a tyramide-based signal amplification approach and visualized by $\mathrm{DAB}$ colorimetric reaction to produce stained slides. Stained slides were scanned on a Huron TissueScope scanner to produce 16-bit tiff images. Sample spots in tiff images were identified and their densities quantified by Array-Pro Analyzer. Relative protein levels for each sample were determined by interpolating each dilution curve 
produced from the densities of the 5-dilution sample spots using a "standard curve" (SuperCurve) for each slide (antibody) [19]. RPPA slide quality was assessed by quality control classifier [20] and only those slides with a quality score above 0.8 were used for further analysis. Differential expression analysis was performed using the LIMMA R package [21], using a Bonferroni corrected LIMMA modified t-test, $\mathrm{p}<0.05$, degree of change is presented as $\log 2$ fold change (LFC).

\section{Results \\ HER2 Overexpressing MCF10A Cells are Sensitive to Olaparib}

To investigate the relationship between HER2 overexpression and sensitivity to PARPi, we used lentivirus-mediate HER2 overexpression to create an isogenic panel of MCF-10A human breast epithelial cells, referred to as MCF-10A-HER2. Isogenic MCF10A-HER2 cell lines were generated as previously described [22]. The resulting clones, exhibited EGFindependent growth, a surrogate marker of cell transformation in MCF-10A cells, indicating that cell growth is driven through HER2 receptor signaling [23]. Further establishing a dependence on the HER2 signaling pathway for proliferation, MCF-10A-HER2 cells were sensitive to two HER2 targeted therapies, trastuzumab and lapatinib, (Figure $1 \mathbf{A}-\mathbf{B}$ ), two-way ANOVA, $\mathrm{n}=5, \mathrm{p}<0.0001)$.

After establishing that MCF-10A-HER2 cells exhibit characteristics of transformed HER2 breast cancer cells, we sought to determine whether these cells are sensitive to the PARP inhibitor olaparib. Olaparib induced a dose-dependent decrease in cell proliferation in two independent MCF-10A-HER2 clones (Figure 1C), Mixed-effects analysis, $n=5, \mathrm{p}<$ 0.0001 .

Trastuzumab and Lapatinib Enhance Olaparib Efficacy in HER2 Overexpressing MCF-10A Cells As both lapatinib and trastuzumab are HER2-targeted therapies, we reasoned that the addition of olaparib to a combination of both trastuzumab and lapatinib may augment their effects in HER2 positive breast cancer cells. To test this hypothesis, we developed a threedrug combination cell viability assay in 96-well plates utilizing a checkerboard design (Supplemental Figure 1). We used a fixed concentration of $13.5 \mathrm{nM}$ for lapatinib across all the treatment conditions, which allowed for 45 distinct drug concentrations of the three drugs $(\mathrm{T}+\mathrm{O}+\mathrm{L}), 9$ concentrations of with olaparib + lapatinib $(\mathrm{O}+\mathrm{L})$, and 5 concentrations with trastuzumab + lapatinib $(\mathrm{T}+\mathrm{L})$. Concentrations of trastuzumab ranged from $(0-0.6 \mu \mathrm{M})$ and olaparib ranged from $(0-10 \mu \mathrm{M})$. Three dimensional graphs were used to visualize the drug combinations effect on cell viability (Figure 2 A and B) Using least squares multiple linear regression, we found that olaparib concentration, trastuzumab concentration, and HER2 status had significant effects on cell viability $(\mathrm{p}<0.0001)$. Comparison of linear regression models including and excluding HER2 status as an independent variable indicated that MCF-10A-HER2 cells were significantly more sensitive to the threedrug combination treatment (Table 1). We further examined the combination of olaparib with trastuzumab and lapatinib to determine if the effects seen in our viability assay were additive or synergistic. Using the synergyfinder $\mathrm{R}$ package, we compared the three drug combination to the expected responses based on the viability of the olaparib + lapatinib and trastuzumab + lapatinib combinations [18]. This allowed us to test for synergy or additive effects using four commonly used reference models: highest single agent (HSA), Bliss independence (Bliss), zero interaction potency (ZIP), and Loewe additivity (Loewe) [24-26]. Bliss independence analysis revealed an average interaction score of 8.72 for MCF10A-HER2 cells, meaning that $8.72 \%$ more cells died when the drugs were used in combination than when adding the individual effects of each drug, which suggests a synergistic effect between olaparib and lapatinib plus trastuzumab. However, parental MCF10A cells had average Bliss interaction score of -4.07 , meaning that $4.07 \%$ less cells died with the combination of drugs than the added contribution of each individual drug (Figure $2 \mathbf{C}$ and D). A one sample t-test was used to confirm that the distribution of drug interactions scores deviated significantly from a zero-centered normal distribution for each reference model (Supplementary Table 2). All drug interaction scores were significant at $\mathrm{p}<0.01$ confirming significant synergistic and antagonistic effects. Consistent across all four reference models, our results show that the three-drug combination was synergistic in MCF-10A-HER2, and slightly antagonistic in parental MCF-10A cells (Supplemental Figure 2, Supplementary Table 3).

\section{Cells with Acquired Resistance to Lapatinib Retain Sensitivity to Olaparib}

We observed that HER2 overexpression sensitizes cells to olaparib, and olaparib synergizes with HER2 targeted therapies. Therefore, we sought to explore the effects of olaparib on HER2-positive breast cancers that are resistant to HER2-directed therapies. To test this hypothesis, we utilized the human breast cancer cell line SKBR3, which is sensitive to both olaparib and lapatinib and we obtained clones of SKBR3 cells that were conditioned to be resistant to lapatinib (SKBR3-LR). We first confirmed that SKBR3-LR cells are resistant to lapatinib compared to parental SKBR3 cells (Figure 3A). After establishing SKBR3-LR cells are resistant to lapatinib, SKBR3-LR and SKBR3 cells were treated with increasing concentrations of olaparib. Olaparib treatment results in a statistically significant decrease 
in growth for both parental and SKBR3-LR cells (Figure 3B).

To identify altered proteins that may be associated with olaparib sensitivity in lapatinib-resistant cells, we turned to reverse phase protein array (RPPA). Of the 306 proteins assayed, 126 proteins (70 decreased and 56 increased) were significantly differentially expressed between lapatinib resistant and parental cells ( $p<0.05$; Supplemental figure 3). In the absence of drug treatment, MCL-1 is upregulated in SKBR3-LR cells, and is a potent inhibitor of apoptosis and cell cycle progression (LFC 0.168; p < 0.05 ; Figure 3C). MCL-1 exerts in anti-apoptotic effect through inhibition of the pro-apoptotic proteins Bim and Bid, which are also significantly upregulated in SKBR3-LR cells (LFC 0.260, 0.151; p $<0.05$; Figure 3C). In addition to MCL-1 upregulation, the MCL-1 associated pro-apoptotic proteins CASP8, and BAX were significantly decreased (LFC of -0.14 , and -0.09 respectively; $\mathrm{p}<$ 0.05; Figure 3C).

To analyze protein changes associated with olaparib treatment, SKBR3-LR and SKBR3 cells were treated with $10 \mu \mathrm{M}$ olaparib for 24 hours. RPPA revealed that upon olaparib treatment, MCL-1 expression was significantly decreased in SKBR3-LR cells (LFC 0.129 ; $<<0.05$; Figure 4D) while expression of Bim and Bid remain similar to DMSO. A reduction in MCL-1 protein expression upon olaparib treatment shifts the balance anti-apoptotic/apoptotic balance in the mitochondria leaving Bim and Bid unabated to induce apoptosis.

\section{Discussion}

The development of HER2 targeted therapies for the treatment of HER2 positive breast cancer is widely regarded as a successful example of personalized medicine and pharmacogenomics. However, acquired resistance to targeted therapy remains a significant obstacle to treatment. Evaluating novel drug combinations is a promising avenue for the development of new treatments for subtypes of breast cancer. Here we show that overexpression of HER2 in non-tumorigenic MCF-10A cells sensitizes cells to olaparib. Consistent with these findings, Yang et al. reported that overexpression of HER2 in MCF7 cells sensitized cells to the PARP inhibitors veliparib and olaparib [9]. In these studies, authors attributed the anti-proliferative activity of the PARP inhibitors to downregulation of NFkB signaling, as a result of inhibition of RELA transcriptional activity. However, a subsequent study of olaparib did not find significant downregulation of NFkB/RELA in the same cell line [10]. In our studies, olaparib treatment in both SKBR3-LR and SKBR3 cells resulted in significantly increased expression of RELA protein. This suggests that NFkB may not be associated with PARP inhibitor sensitive in HER-positive breast cancers.
However, it is important to note that the mechanism by which HER2 expression sensitizes cells to PARPi is not conclusively understood. One mechanism by which HER2 expression sensitizes cells to olaparib may be related to proliferative stress, as HER2 overexpression is known to significantly increase proliferation rates and subsequently increase proliferative stress, which results in sensitivity to PARP trapping and DNA damage repair inhibition $[6,27]$. Studies on the direct effects of HER2 and PARP1 have found that expression of HER2 increases expression of PARP1 [28], possibly through reduced expression of the miRNA let-7a [29].

Lapatinib has previously been shown to increase the efficacy of olaparib in both HER2 overexpressing and non-HER2 overexpressing cancer cells, through impaired homologous recombination efficiency with lapatinib treatment [30]. Trastuzumab has also been reported to increase the efficacy of olaparib through downregulation of PCNA, a protein involved in HR, in HER2 overexpressing cancer cells [10]. In line with these reports, we show that olaparib enhanced the efficacy of trastuzumab and lapatinib combination therapy, and that these drugs act in synergy in HER2 overexpressing MCF10A cells. The addition of trastuzumab to lapatinib and olaparib containing therapy induced significant synergistic cell killing. Both of these drugs have been shown to downregulate HR [10, 30]; however, Yang et al. has shown that the effect of single agent olaparib on HER2-expressing cells is unlikely due to a deficiency in HR repair. [9] It may be possible that the mechanism by which olaparib exerts its anti-cancer activity may differ when given as a single agent vs. in combination with trastuzumab and lapatinib. This question may be answered by the OPHILIA clinical trial, which is currently investigating the safety and efficacy of olaparib in combination with trastuzumab in HR deficient advanced breast cancers. The results of this trial greatly impact our understanding of the clinical utility of olaparib in combination with trastuzumab in the treatment of HR deficient HER2 positive breast cancer. Outside the context of HR deficient breast cancer, the synergistic effects seen when these drugs are used in combination suggests that a similar mechanism of action or pathway is being targeted, but further mechanistic evaluation is needed to determine the extent to which the HR pathway is involved.

Acquired resistance to lapatinib has been reported to involve significant molecular reprogramming of cell signaling [31-35], and modulation of the PI3K/MAPK pathways [36], which are major targets of trastuzumab and lapatinib therapies. In our studies, olaparib displayed significant synergy when combined with trastuzumab and lapatinib. Taken 
together, we hypothesized that lapatinib resistance may affect the response of olaparib treatment. Surprisingly, in our SKBR3 model of acquired resistance to lapatinib, we found these cells retain their sensitivity to olaparib, albeit to a lesser extent than parental cells. RPPA analysis of SKBR3-LR cells revealed upregulation of MCL-1 and its proapoptotic targets Bim and Bid, when compared to parental SKBR3 cells. HER2 breast cancers express elevated levels of MCL-1 [37], and upregulation of MCL-1 has previously been shown to be a mechanism of acquired resistance to lapatinib [3840]. MCL-1 exerts its anti-apoptotic function by binding and sequestering $\mathrm{BH} 3$ proteins in the mitochondria, such as Bim and Bid, and preventing their activation of BAX and BAK. Bim expression has been shown to be upregulated in HER2 breast cancer cells in a c-Myc dependent manner, and requires the anti-apoptotic function of MCL-1 to prevent apoptosis [41]. Upon olaparib treatment of SKBR3-LR cells, MCL-1 protein expression is dramatically decreased, while expression of Bim and Bid are only slightly decreased and not statistically different from DMSO treatment. Unabated expression of $\mathrm{Bim}$ and $\mathrm{Bid}$ in the mitochondria following olaparib treatment may induce apoptosis in SKBR3-LR cells. Importantly, targeted inhibition of MCL-1 has been shown to result in induction of apoptosis in a Bim dependent manner [40-42]. Without expression of pro-apoptotic $\mathrm{BH} 3$ only proteins in the mitochondria, inhibition of MCL-1 is unlikely to induce apoptosis. Priming cells for apoptosis through the induction of BH3-only proteins have proven to be an effective strategy for combination treatments with MCL-1 inhibitors [43]. The MCL-1 inhibitor S63845 has been shown to synergize with Trastuzumab and Lapatinib in HER2 positive breast cancer cells, by priming cells for apoptosis through the induction of Bim expression $[40,44]$. However, it remains unclear to what extent downregulation of MCL-1 has on the mechanism by which HER2 overexpression sensitizes cells to PARPi.

Acquired resistance to lapatinib has been shown to be heterogeneous in nature and involves a myriad of pathways and can vary across cell lines [31, 36]. Supporting our observations, veliparib, another PARP inhibitor, was reported to induce apoptosis in a three cell line models of trastuzumab resistance [45]. Our results provide evidence to support the continued research into the effects of single-agent PARPi or in combination with HER2 targeted therapies for the treatment of HER2 positive breast cancers with acquired resistance to HER2-targeted therapies.
A

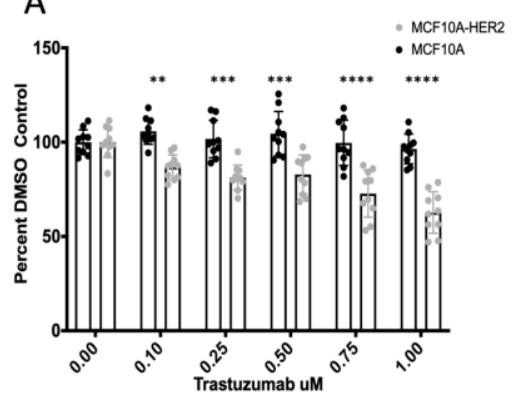

C

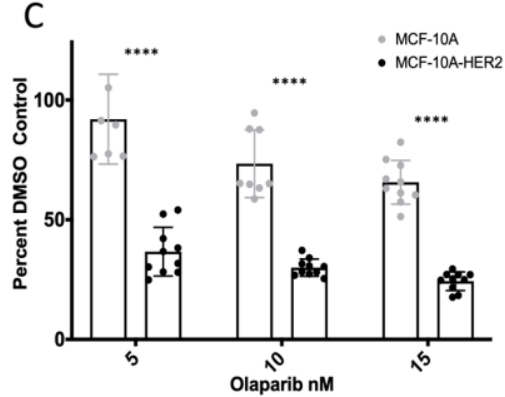

B

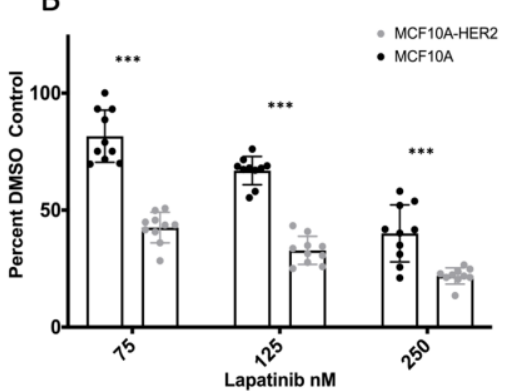

Figure 1. HER2 overexpression induces sensitivity to olaparib in MCF-10A cells.

(A) MCF-10A-HER2 and MCF10A cells cultured in increasing concentrations of trastuzumab, analyzed by two-way ANOVA with Bonferroni multiple comparisons test, $\mathrm{n}=5, \mathrm{p}<0.0001$. (B) MCF-10A-HER2 and MCF10A cells cultured in increasing concentrations of lapatinib, analyzed by two-way ANOVA with Bonferroni multiple comparisons test, $n=5, p<0.0001$. (C) MCF-10A-HER2 and MCF10A cells cultured in increasing concentrations of olaparib, analyzed by Mixed Effects analysis with Bonferroni multiple comparisons test, $n=5, p<0.0001$. (ns $=p>$ $0.05, *=\mathrm{p}<0.05, * *=\mathrm{p}<0.01, * * *=\mathrm{p}<0.001, * * * * \mathrm{p}<0.0001)$. 
A MCF10A Trastuzumab-Lapatinib-Olaparib Combination

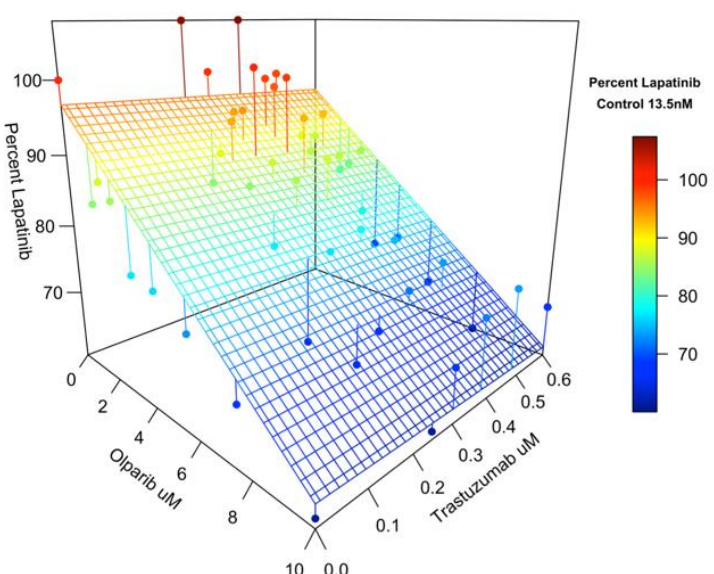

B MCF10A-HER2 Trastuzumab-Lapatinib-Olaparib Combination

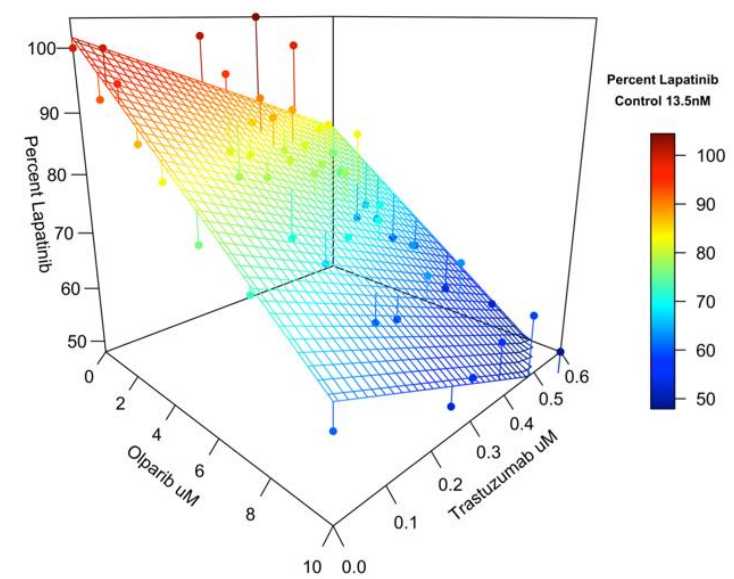

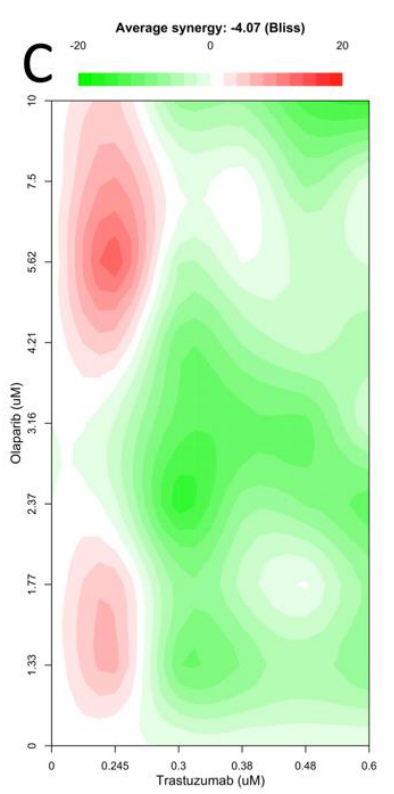
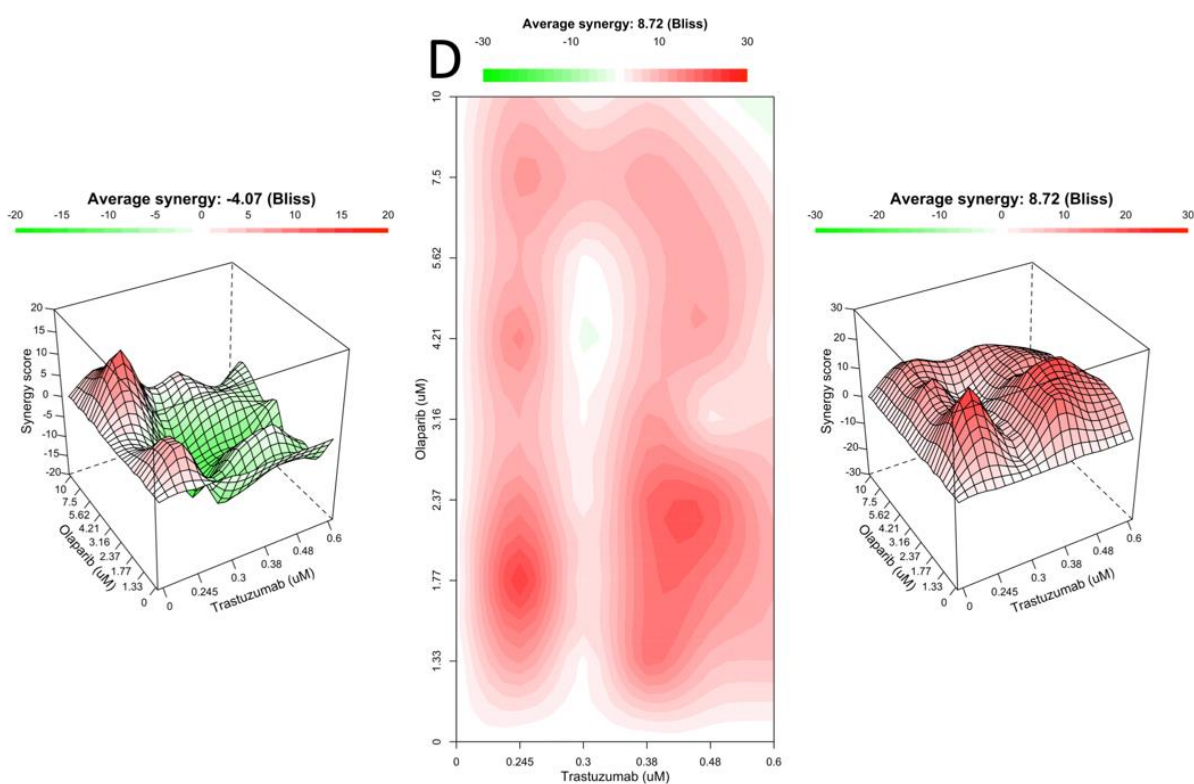

Figure 2. Trastuzumab-lapatinib-olaparib combination drug treatment induce synergistic cytotoxicity in HER2 expressing MCF10A cells. (A) Three dimensional graph depicting cell viability of MCF10A cells in response to trastuzumab-lapatinib-olaparib combination drug treatments. (B) Three dimensional graph depicting cell viability of MCF10A-HER2 cells in response to trastuzumab-lapatinib-olaparib combination drug treatments. Cell viability for combination treatment is normalized to a $13.5 \mathrm{nM}$ lapatinib control. (A-B)Concentrations of trastuzumab increase across the $\mathrm{x}$ axis, while concentrations of olaparib increase along the $\mathrm{y}$ axis, cell viability is plotted along the $\mathrm{z}$ axis. A generalized linear model was used to generate a surface depicting overall best fit values for response to treatment. (C) Bliss independence drug interaction scores for MCF10A cells response to trastuzumab-lapatinibolaparib combination drug treatment. (D) Bliss independence drug interaction scores for MCF10A-HER2 cells response to trastuzumab-lapatinib-olaparib combination drug treatment. (C-D) Positive scores (red) indicate drug synergy, negative scores (green) indicate drug antagonism, and scores close to zero (white) indicate additivity. Concentrations of olaparib increase along the vertical axis while concentrations of trastuzumab increase along the horizontal axis - lapatinib concentration was held constant at $13.5 \mathrm{nM}$ for all wells. Drug interaction scores were calculated using cell viability across 5 plates ( $\mathrm{n}=5$ for each concentration). Drug interaction scores were averaged across all concentrations to yield a single averaged score. 
A
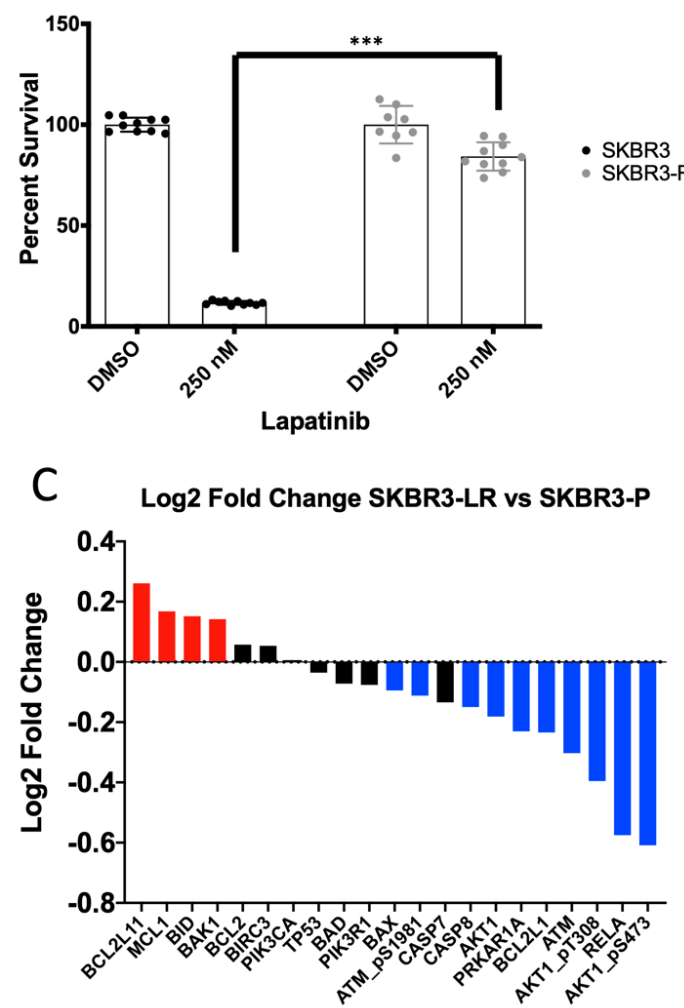

B

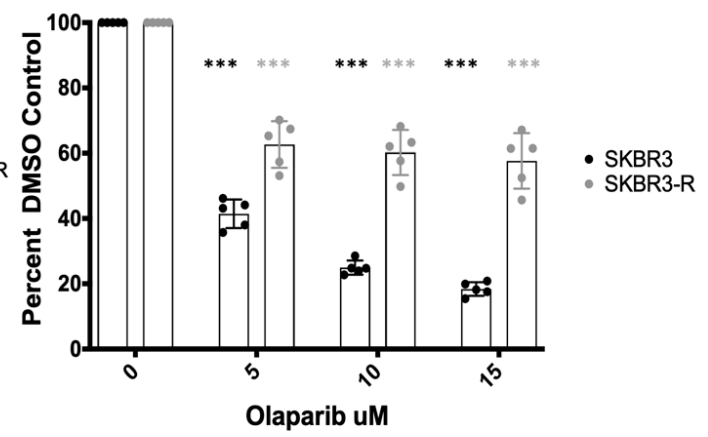

D Differential Protein Expression SKBR3-LR Olaparib vs DMSO

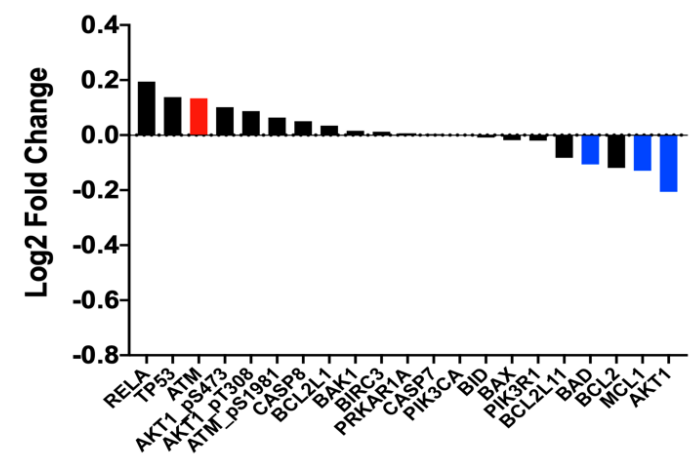

Figure 3. Olaparib is cytotoxic in lapatinib resistant SKBR3 cells through downregulation of MCL-1. (A) SKBR3 and SKBR3-LR cells cultured in DMSO or 250nM lapatinib, analyzed by Mixed Effects Analysis with Bonferroni multiple comparisons test, $\mathrm{n}=10, \mathrm{p}<0.0001$. (B) SKBR3-LR and SKBR3 cells culture in increasing concentrations of olaparib, analyzed by two-way ANOVA with Bonferroni multiple comparisons test, $\mathrm{n}=5$, $\mathrm{p}<0.0001$. (C) Differential protein expression of RPPA data for SKBR3-LR cells compared with parental SKBR3 cells for proteins associated with MCL-1 regulation of intrinsic apoptosis, LIMMA modified t-test and Bonferroni multiple comparisons correction (red $=$ upregulated $\mathrm{p}<0.05$, black $=\mathrm{ns}$, and blue $=$ downregulated $\mathrm{p}<0.05$ ). (D) Differential protein expression of RPPA data for SKBR3-LR cells treated with olaparib compared with DMSO vehicle control for proteins associated with MCL-1 regulation of intrinsic apoptosis, LIMMA modified t-test and Bonferroni multiple comparisons correction ( $\mathrm{red}=$ upregulated $\mathrm{p}<0.05$, black $=\mathrm{ns}$, and blue $=$ downregulated $\mathrm{p}<0.05)$.

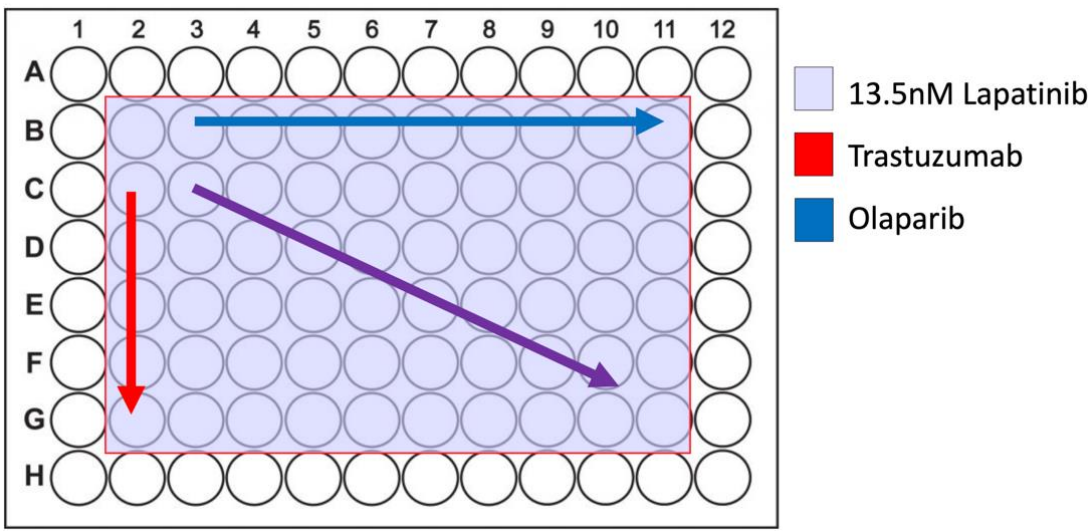

Supplemental Figure 1. Trastuzumab-lapatinib-olaparib combination drug assay checkerboard design. Using only the inside 60 wells of a 96 well plate, $13.5 \mathrm{~nm}$ Lapatinib remained constant across all of the treatment combination (purple), while concentrations of trastuzumab (red) increased across rows, and olaparib (blue) increased along columns. Trastuzumab concentration ranged from 0-0.6uM and olaparib concentration ranged from 0-10uM. Utilizing this checkerboard design results in 59 unique concentrations of the three drugs. 


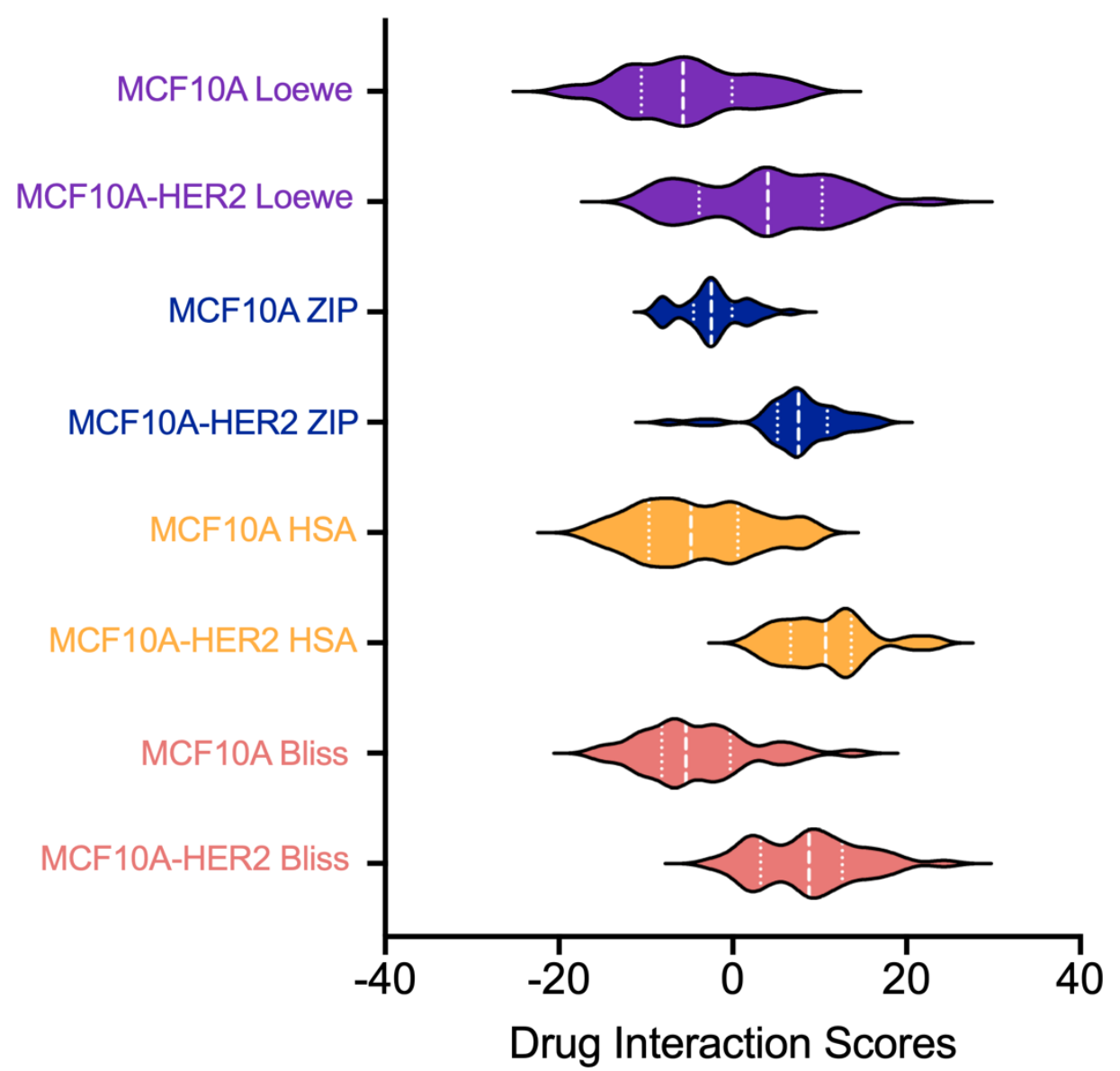

Supplemental Figure 2. Distribution of drug interaction scores in MCF10A and MCF10A-HER2 cells. Drug interaction scores were calculated using four commonly used reference models; Bliss independence (Bliss), highest single agent (HSA), Loewe additivity (Loewe), and zero interaction potency (ZIP) for MCF10A and MCF10AHER2 cells. Interaction scores for every drug concentration $(n=40)$ were plotted using Graphpad Prism 8. Violin plots depict the distribution of scores across all concentrations for each cell line and reference model. Positive scores indicate drug synergy, negative scores indicate drug antagonism, and scores close to zero indicate additivity. White dashed lines indicate median and quartiles. 
A
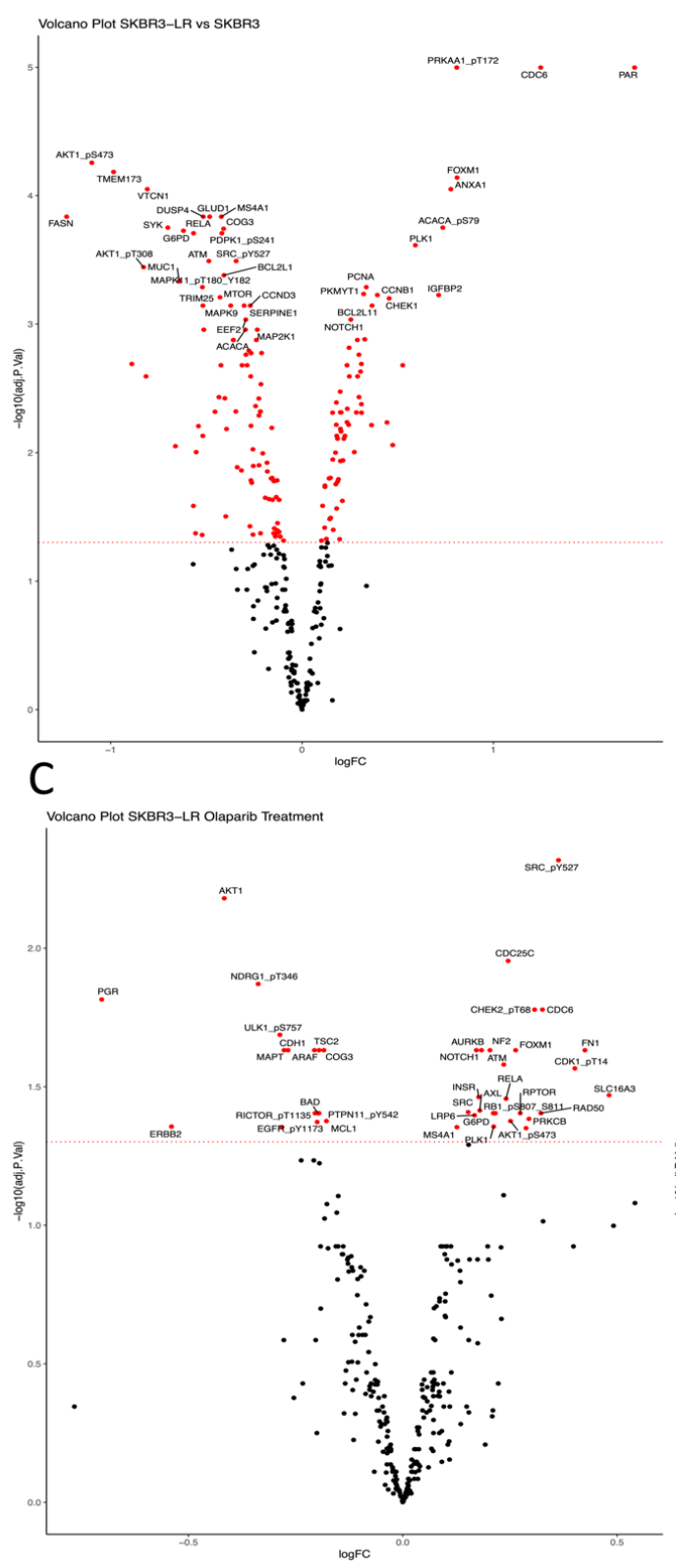

B

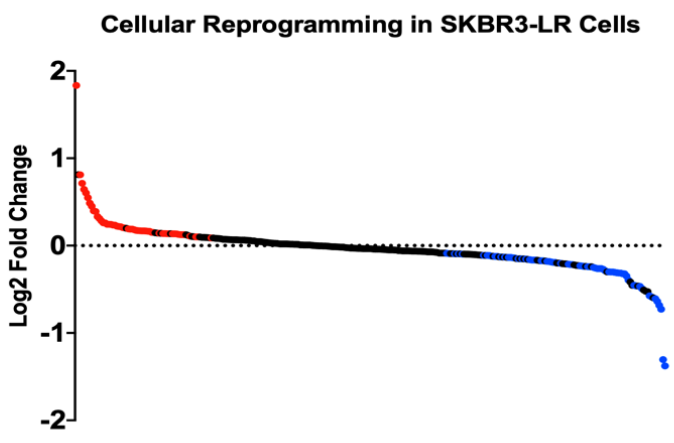

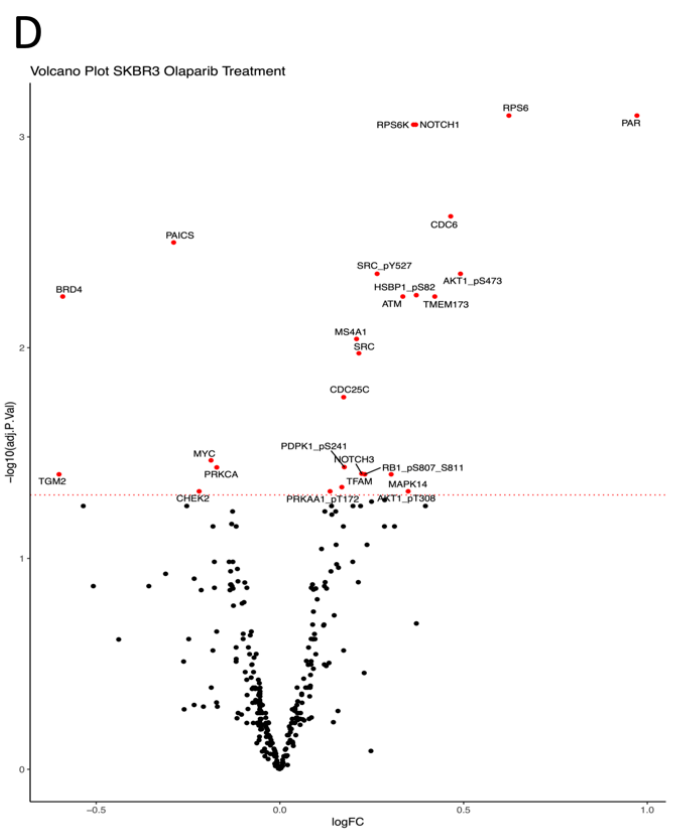

Supplemental Figure 3. Global protein changes associated with lapatinib resistance in SKBR3-LR cells. Reverse phase protein array data from SKBR3-LR and SKBR3 cells was used to determine differential protein expression associated with lapatinib resistance. (A) Differential protein expression of 306 proteins analyzed by RPPA analysis, significance was determined using the LIMMA modified t-test and Bonferroni multiple comparisons correction (red $=$ upregulated $\mathrm{p}<0.05$, black $=\mathrm{ns}$, and blue $=$ downregulated $\mathrm{p}<0.05$ ). (B) Volcano plot depicting differential protein expression between SKBR3-LR and SKBR3 cells, significance was determined using the LIMMA modified t-test and Bonferroni multiple comparisons correction (red $=p<0.05$ ). (C) Volcano plot depicting differential protein expression between SKBR3-LR cells treated with DMSO control and SKBR3-LR cells treated with $10 \mu \mathrm{M}$ olaparib, significance was determined using the LIMMA modified t-test and Bonferroni multiple comparisons correction ( $\mathrm{red}=\mathrm{p}<0.05$ ). (D) Volcano plot depicting differential protein expression between SKBR3LR cells treated with $10 \mu \mathrm{M}$ olaparib and SKBR3 cells treated with $10 \mu \mathrm{M}$ olaparib. Protein expression was baseline corrected to account for differences basal protein expression between the two cell lines. This comparison will test for proteins that response differently between the two cell lines upon olaparib treatment. Significance was determined using the LIMMA modified t-test and Bonferroni multiple comparisons correction $($ red $=p<0.05)$. 


\begin{tabular}{|c|c|c|c|c|c|}
\hline Parameter Estimates & & & & & \\
\hline Variable & Model & Intercept & Olaparib [ ] & Trastuzumab [ ] & HER2 Status \\
\hline \multirow{2}{*}{ Estimate } & Model 1 & 102.7 & -3.547 & -18.56 & -7.117 \\
\hline & Model 2 & 99.17 & -3.547 & -18.56 & \\
\hline \multirow{2}{*}{ Standard error } & Model 1 & 1.854 & 0.2339 & 3.739 & 1.415 \\
\hline & Model 2 & 1.901 & 0.2595 & 4.149 & \\
\hline \multirow{2}{*}{$95 \%$ confidence interval } & Model 1 & 99.06 to 106.4 & -4.011 to -3.083 & -25.98 to -11.15 & -9.923 to -4.311 \\
\hline & Model 2 & 95.41 to 102.9 & -4.062 to -3.032 & -26.79 to -10.34 & \\
\hline \multicolumn{6}{|l|}{ Goodness of Fit } \\
\hline Model & Multiple R & Adjusted R squared & AICc & & \\
\hline Model 1 & 0.8539 & 0.7213 & 437.5 & & \\
\hline Model 2 & 0.8144 & 0.6633 & 458.8 & & \\
\hline \multicolumn{6}{|l|}{ Comparison of Models } \\
\hline Null hypothesis & Model 2 & & & & \\
\hline Alternative hypothesis & Model 1 & & & & \\
\hline Preferred model & Model 1 & & & & \\
\hline Conclusion (alpha $=0.05$ ) & Reject null hypothesis & & & & \\
\hline P value & $P<0.0001$ & & & & \\
\hline$F(D F n, D F d)$ & $25.29(1,104)$ & & & & \\
\hline
\end{tabular}

Table 1. Least squares multiple linear regression analysis of trastuzumab-lapatinib-olaparib combination drug assay. Model 1 was fit including HER2 status as a variable, while model 2 did not include HER2 status. The table include estimated coefficients for each parameter, goodness of fit for each model, and a comparison of the two models to test the null hypothesis that HER2 status has no effect on cell viability in response to combination drug treatment.

\begin{tabular}{|c|c|c|c|c|c|c|c|c|}
\hline Cell Line & MCF10A-HER2 & MCF10A & MCF10A-HER2 & MCF10A & MCF10A-HER2 & MCF10A & MCF10A-HER2 & MCF10A \\
\hline Drug Interaction Method & \multicolumn{2}{|c|}{ Bliss } & \multicolumn{2}{|c|}{ HSA } & \multicolumn{2}{|c|}{ ZIP } & \multicolumn{2}{|c|}{ Loewe } \\
\hline Theoretical mean & 0 & 0 & 0 & 0 & 0 & 0 & 0 & 0 \\
\hline Actual mean & 8.718 & -4.07 & 10.73 & -4.05 & 7.714 & -2.339 & 3.799 & -5.336 \\
\hline Number of values & 40 & 40 & 40 & 40 & 40 & 40 & 40 & 40 \\
\hline$t, d f$ & $\mathrm{t}=9.034, \mathrm{df}=39$ & $t=3.991, d f=39$ & $\mathrm{t}=12.82, \mathrm{df}=39$ & $t=3.728, d f=39$ & $t=9.982, d f=39$ & $t=4.036, d f=39$ & $\mathrm{t}=2.948, \mathrm{df}=39$ & $\mathrm{t}=4.933, \mathrm{df}=39$ \\
\hline P value (two tailed) & $<0.0001$ & 0.0003 & $<0.0001$ & 0.0006 & $<0.0001$ & 0.0002 & 0.0054 & $<0.0001$ \\
\hline Discrepancy & 8.718 & -4.07 & 10.73 & -4.05 & 7.714 & -2.339 & 3.799 & -5.336 \\
\hline SD of discrepancy & 6.104 & 6.451 & 5.293 & 6.871 & 4.888 & 3.665 & 8.151 & 6.842 \\
\hline SEM of discrepancy & 0.9651 & 1.02 & 0.8369 & 1.086 & 0.7728 & 0.5796 & 1.289 & 1.082 \\
\hline 95\% confidence interval & 6.76 to 10.67 & -6.13 to -2.00 & 9.03 to 12.42 & -6.24 to -1.85 & 6.15 to 9.27 & \begin{tabular}{|l|}
-3.51 to -1.16 \\
\end{tabular} & 1.19 to 6.40 & -7.52 to -3.14 \\
\hline R squared (partial eta squared) & 0.6766 & 0.29 & 0.8082 & 0.2627 & 0.7187 & 0.2946 & 0.1822 & 0.3842 \\
\hline
\end{tabular}

Supplementary Table 1. One sample t-test of drug interaction scores. All samples passed Kolmogorov-Smirnov normality at $\mathrm{p}>0.05$.

\begin{tabular}{|c|c|c|c|c|c|c|c|c|}
\hline Cell Line & \multicolumn{4}{|c|}{ MCF10A } & \multicolumn{4}{c|}{ MCF10A-HER2 } \\
\hline Model & Bliss & HSA & ZIP & Loewe & Bliss & HSA & ZIP & Loewe \\
\hline Mean & -4.07 & -4.05 & -2.34 & -5.34 & 8.72 & 10.73 & 7.71 & 3.80 \\
\hline SEM & 1.02 & 1.09 & 0.58 & 1.08 & 0.97 & 0.84 & 0.77 & 1.29 \\
\hline Lower 95\% CI & -6.13 & -6.25 & -3.51 & -7.53 & 6.77 & 9.04 & 6.15 & 1.19 \\
\hline Upper 95\% CI & -2.01 & -1.85 & -1.17 & -3.15 & 10.67 & 12.42 & 9.28 & 6.41 \\
\hline
\end{tabular}

Supplementary Table 2. Descriptive statistics of drug interactions by commonly used reference models. Interaction scores were input into Graphpad Prism 8 and analyzed for descriptive statistics.

\section{References}

1. Arteaga, C.L., et al., Treatment of HER2-positive breast cancer: current status and future perspectives. Nat Rev Clin Oncol, 2011. 9(1): p. 16-32.

2. Karunagaran, D., et al., ErbB-2 is a common auxiliary subunit of NDF and EGF receptors: implications for breast cancer. EMBO J, 1996. 15(2): p. 254-64.

3. Vernieri, C., et al., Resistance mechanisms to anti-HER2 therapies in HER2-positive breast cancer: Current knowledge, new research directions and therapeutic perspectives. Crit Rev Oncol Hematol, 2019. 139: p. 53-66.

4. Mohd Sharial, M.S., J. Crown, and B.T. Hennessy, Overcoming resistance and restoring sensitivity to HER2 targeted therapies in breast cancer. Ann Oncol, 2012. 23(12): p. 3007-16.

5. Ray Chaudhuri, A. and A. Nussenzweig, The multifaceted roles of PARP1 in DNA repair and chromatin remodelling. Nat Rev Mol Cell Biol, 2017. 18(10): p. 610-621.

6. Yi, M., et al., Advances and perspectives of PARP inhibitors. Exp Hematol Oncol, 2019. 8: p. 29 
7. Shen, Y., M. Aoyagi-Scharber, and B. Wang, Trapping Poly(ADP-Ribose) Polymerase. J Pharmacol Exp Ther, 2015. 353(3): p. 446-57.

8. Ellsworth, R.E., et al., Amplification of HER2 is a marker for global genomic instability. BMC Cancer, 2008. 8: p. 297.

9. Nowsheen, S., et al., HER2 overexpression renders human breast cancers sensitive to PARP inhibition independently of any defect in homologous recombination DNA repair. Cancer Res, 2012. 72(18): p. 4796-806.

10. Garcia-Parra, J., et al., Poly (ADP-ribose) polymerase inhibition enhances trastuzumab antitumour activity in HER2 overexpressing breast cancer. Eur J Cancer, 2014. 50(15): p. 2725-34.

11. Shimo, T., et al., Antitumor and anticancer stem cell activity of a poly ADP-ribose polymerase inhibitor olaparib in breast cancer cells. Breast Cancer, 2014. 21(1): p. 75-85.

12. Tang, Y., et al., Poly(ADP-ribose) polymerase 1 modulates the lethality of CHK1 inhibitors in mammary tumors. Mol Pharmacol, 2012. 82(2): p. 322-32.

13. Pierce, A., et al., Comparative antiproliferative effects of iniparib and olaparib on a panel of triple-negative and nontriple-negative breast cancer cell lines. Cancer Biol Ther, 2013. 14(6): p. 537-45

14. MedSIR, Olaparib+Trastuzumab in HER2 + , Breast Cancer Susceptibility Gene (BRCA) Mutated or Homologous Recombination Deficient (HRD) Advanced Breast Cancer. 2019, https://ClinicalTrials.gov/show/NCT03931551.

15. Turturro, S.B., et al., Somatic loss of PIK3RI may sensitize breast cancer to inhibitors of the MAPK pathway. Breast Cancer Res Treat, 2019. 177(2): p. 325-333.

16. Soetaert, K., plot3D: Plotting Multi-Dimensional Data. 2017.

17. Team, R.C., R: A Language and Environment for Statistical Computing. 2018, R Foundation for Statistical Computing

18. Ianevski, A., et al., SynergyFinder: a web application for analyzing drug combination dose-response matrix data. Bioinformatics, 2017. 33(15): p. 2413-2415.

19. Hu, J., et al., Non-parametric quantification of protein lysate arrays. Bioinformatics, 2007. 23(15): p. 1986-94.

20. Ju, Z., et al., Development of a robust classifier for quality control of reverse-phase protein arrays. Bioinformatics, 2015. 31(6): p. 912-8.

21. Ritchie, M.E., et al., limma powers differential expression analyses for RNA-sequencing and microarray studies. Nucleic Acids Res, 2015. 43(7): p. e47.

22. DiScala, M., et al., Loss of STAT6 leads to anchorageindependent growth and trastuzumab resistance in HER2+ breast cancer cells. PLoS One, 2020. 15(6): p. e0234146.

23. Gustin, J.P., et al., Knockin of mutant PIK3CA activates multiple oncogenic pathways. Proc Natl Acad Sci U S A, 2009. 106(8): p. 2835-40

24. Yadav, B., et al., Searching for Drug Synergy in Complex Dose-Response Landscapes Using an Interaction Potency Model. Comput Struct Biotechnol J, 2015. 13: p. 504-13.

25. C.I., B., The toxicity of poisons applied jointly. Annals of Applied Biology, 1939. 26: p. 585-615.

26. Berenbaum, M.C., What is synergy? Pharmacol Rev, 1989. 41(2): p. 93-141.

27. Pilie, P.G., et al., PARP Inhibitors: Extending Benefit Beyond BRCA-Mutant Cancers. Clin Cancer Res, 2019. 25(13): p. 3759-3771.
28. Stanley, J., et al., PARP1 and phospho-p65 protein expression is increased in human HER2-positive breast cancers. Breast Cancer Res Treat, 2015. 150(3): p. 569-79.

29. Wielgos, M.E., et al., Let-7 Status Is Crucial for PARPI Expression in HER2-Overexpressing Breast Tumors. Mol Cancer Res, 2017. 15(3): p. 340-347.

30. Nowsheen, S., et al., Synthetic lethal interactions between EGFR and PARP inhibition in human triple negative breast cancer cells. PLoS One, 2012. 7(10): p. e46614.

31. D'Amato, V., et al., Mechanisms of lapatinib resistance in HER2-driven breast cancer. Cancer Treat Rev, 2015. 41(10): p. 877-83

32. Hubalek, M., et al., Resistance to HER2-targeted therapy: mechanisms of trastuzumab resistance and possible strategies to overcome unresponsiveness to treatment. Wien Med Wochenschr, 2010. 160(19-20): p. 506-12.

33. Liu, L., et al., Novel mechanism of lapatinib resistance in HER2-positive breast tumor cells: activation of AXL. Cancer Res, 2009. 69(17): p. 6871-8.

34. Pohlmann, P.R., I.A. Mayer, and R. Mernaugh, Resistance to Trastuzumab in Breast Cancer. Clin Cancer Res, 2009. 15(24): p. 7479-7491.

35. Shi, H., et al., Lapatinib resistance in HER2+ cancers: latest findings and new concepts on molecular mechanisms. Tumour Biol, 2016.

36. Ruprecht, B., et al., Lapatinib Resistance in Breast Cancer Cells Is Accompanied by Phosphorylation-Mediated Reprogramming of Glycolysis. Cancer Res, 2017. 77(8): p. 1842-1853.

37. Campbell, K.J., et al., MCL-1 is a prognostic indicator and drug target in breast cancer. Cell Death Dis, 2018. 9(2): p. 19

38. Martin, A.P., et al., Inhibition of MCL-1 enhances lapatinib toxicity and overcomes lapatinib resistance via BAK dependent autophagy. Cancer Biol Ther, 2009. 8(21): p. 2084-96.

39. Eustace, A.J., et al., Development of acquired resistance to lapatinib may sensitise HER2-positive breast cancer cells to apoptosis induction by obatoclax and TRAIL. BMC Cancer, 2018. 18(1): p. 965.

40. Bashari, M.H., et al., Mcl-1 confers protection of Her2 positive breast cancer cells to hypoxia: therapeutic implications. Breast Cancer Res, 2016. 18(1): p. 26.

41. Campone, M., et al., $c-M y c$ dependent expression of proapoptotic Bim renders HER2-overexpressing breast cancer cells dependent on anti-apoptotic Mcl-1. Mol Cancer, 2011. 10: p. 110.

42. Williams, M.M., et al., Therapeutic inhibition of Mcl-1 blocks cell survival in estrogen receptor-positive breast cancers. Oncotarget, 2019. 10(52): p. 5389-5402.

43. Hird, A.W. and A.E. Tron, Recent advances in the development of Mcl-1 inhibitors for cancer therapy. Pharmacol Ther, 2019. 198: p. 59-67.

44. Merino, D., et al., Synergistic action of the MCL-1 inhibito S63845 with current therapies in preclinical models of triplenegative and HER2-amplified breast cancer. Sci Transl Med, 2017. 9(401).

45. Wielgos, M.E., et al., Trastuzumab-Resistant HER2(+) Breast Cancer Cells Retain Sensitivity to Poly (ADP-Ribose) Polymerase (PARP) Inhibition. Mol Cancer Ther, 2018. 17(5): $\quad$ p. $921-930$ 